\title{
Condensate fluctuations of a trapped, ideal Bose gas
}

\author{
H. David Politzer* \\ California Institute of Technology, Pasadena, California 91125
}

(Received 11 June 1996)

\begin{abstract}
For a non-self-interacting Bose gas with a fixed, large number of particles confined to a trap, as the ground-state occupation becomes macroscopic, the condensate number fluctuations remain microscopic. However, this is the only significant aspect in which the grand canonical description differs from canonical or microcanonical in the thermodynamic limit. General arguments and estimates including some of the vanishingly small quantities are compared to explicit, fixed-number calculations for $10^{2}-10^{6}$ particles. [S1050-2947(96)06312-3]
\end{abstract}

PACS number(s): 03.75.Fi, 05.30.Jp, 05.40.+j, 05.70.Fh

\section{INTRODUCTION}

Large fluctuations are a salient feature of the thermal behavior of systems of bosons. For example, if $n$ is the mean number of noninteracting particles occupying a particular one-particle state, then the mean-square occupation fluctuation is $n(n+1)$. This is easily derived in the grand canonical picture by considering diffusive equilibrium with a particle reservoir characterized by a chemical potential [1]. If, however, the system has a fixed total number of particles $N$ confined in space by a trapping potential or container, then at low enough temperature $T$ or fixed total energy $E$ when a significant fraction of $N$ are in the ground state, such large fluctuations are impossible. No matter how large $N$, this aspect of the grand canonical description cannot be even approximately true. This paper addresses what does happen for fixed large $N$ as $N \rightarrow \infty$.

A decades-old answer to this question is that any interaction between the particles would eliminate such large fluctuations, even in the presence of a chemical potential. With a weak interparticle interaction and a chemical potential, fluctuations in the occupations of various states are only weakly correlated. Therefore, the fluctuation in the total number of particles not in the ground state is microscopic. Hence, a macroscopic condensate fluctuation would mean a macroscopic density fluctuation. Even if the particles interact weakly, this would mean a macroscopic energy fluctuation. The consequent macroscopic rise in free energy would suppress the fluctuation. (See Appendix B for a more formal sketch of this argument.) Thus, with interactions producing a finite compressibility, the equivalence of the three standard statistical ensembles is assured in the thermodynamic limit, and the computationally convenient chemical potential can still be used for isolated, large systems [2]. In the context of Bose liquids, the ideal gas is a theoretical curiosity. Large condensate fluctuation is only one of several features for which ignoring interactions gives qualitatively incorrect results [3].

This argument does not address the question of what does happen to condensate fluctuations of the ideal Bose gas. Furthermore, this is not a totally idle or purely theoretical ques-

*Electronic address: politzer@theory.caltech.edu tion. In current experimental work on the trapping and cooling of bosonic atoms, there is typically no diffusive particle or thermal energy reservoir [4-6]. While the atoms most certainly interact, $N \neq \infty$. Hence, one can ask about the system as a whole rather than only describing densities (intensive quantities), which are really just subvolumes in diffusive and thermal equilibrium with their (much larger) surroundings. For subvolumes of an infinite system, $\mu$ and $T$ give an appropriate description. However, for a finite, isolated system taken as a whole, which has a greater impact on the condensate fluctuations, the particle interactions or the constraint of fixed total $N$ ? The answer depends on the density realized in the particular situation. A practical distinction of a gas from a liquid is that the density can be easily varied over many orders of magnitude. In the first successful experiments [4], there are noticeable effects of interparticle repulsion; and many of the more detailed observations currently underway require a mean-field (albeit weak) description of the interparticle scattering length to reconcile theory with observations. Nevertheless, it is possible to imagine approaching Bose condensation with a box or trap so large and density so low that the effects of a given interatomic interaction, characterized by a fixed scattering length, are negligible, even for density fluctuations of the order of the equilibrium density. (An estimate of the requisite relation of the scattering length, trap parameters, and density is given in Appendix B.) Even though the Bose-Einstein transition temperature decreases with decreasing density, the total energy shift due to a weak fixed-strength interparticle interaction decreases faster. Also, the actual interatomic interactions may not serve to stabilize anything. Rather, the gaseous state may itself only be metastable [5]. In such situations, the equilibrium statistics of the ideal gas are certainly a better starting approximation than the equilibrium statistics of the interacting system.

After a summary of a variety of potentially confusing issues (Sec. II), a thoroughly elementary analysis of the problem (Sec. III) suggests that the condensate fractional fluctuations vanish with increasing $N$, but all other significant grand canonical predictions have vanishing corrections. This is also sufficient to establish the equivalence of using either fixed $T$ or fixed total $E$ to characterize the system for large $N$. The proposed picture provides an explicit prediction (Sec. IV) for the condensate fluctuation as well as the values of observ- 
ables, e.g., two-level correlations, that are identically zero with a chemical potential but are induced by fixing $N$. (With a natural normalization, such functions are vanishingly small as $N \rightarrow \infty$.) The results of a numerical evaluation of the canonical partition function and related functions for $N$ from $10^{2}$ to $10^{6}$ (Sec. V) confirm these predictions. Some obvious conclusions are offered (Sec. VI), while comments on details of the numerical work are left to Appendix A. Appendix B outlines the simple estimate of the condensate fluctuation damping due to repulsive interactions, which allows a comparison with the effect due to fixing $N$.

\section{POTENTIAL ISSUES}

It is only the noninteracting particles in the ground state of a trap or confining potential that do not satisfy the hypotheses of the standard demonstration [2] of the equivalence of the grand canonical and canonical ensembles in the thermodynamic limit. Hence, the questions raised here only arise if the ground-state occupation is macroscopic. At ultralow $T$ when almost all particles are in the ground state, the condensate serves as a particle reservoir for all the excited states, and so some form of the grand canonical description for excited states should be valid in that domain. But what about intermediate $T$ 's? Is the inequivalence of chemical potential and fixed $N$ limited to the size of the ground-state fluctuations? If the condensate manifested the boson propensity for large fluctuations and there were any macroscopic fluctuation in the condensate number, it would have to be accompanied by correlations between the various occupation numbers. (Such correlations are identically zero for the grand canonical ideal gas.) There need not be any macroscopic fluctuation in the average density because the total number is fixed. Yet, larger than anticipated excited-state fluctuations and correlations might lead to larger fluctuations in the total $E$ at fixed $T$. And were this the case, the equivalence of fixing $E$ and fixing $T$ might be lost in the thermodynamic limit.

Chemical potential is not just a calculational convenience. There is really no practical alternative for analytic calculations because not much is known directly about the large but fixed $N$ asymptotics of the canonical or microcanonical partition functions, even for systems as simple as the ideal Bose gas. If this analytic tool were lost, theory would be reduced almost entirely to numerical techniques.

\section{FIXED- $N$ STATISTICS}

The resolution of these conundrums lies in the observation that the grand canonical excited-state occupations in the thermodynamic limit are independent of not only the condensate fluctuations but the condensate occupation itself. Hence, if the behavior of the excited-state occupancies can be reliably estimated using the concept of a chemical potential, one can deduce the behavior of the condensate from the constraint of fixed $N$. This argument is really just a minor extension of the traditional one used to compute the condensate fraction $[7,1]$. In particular, it goes as follows.

Let $i$ label the one-particle (or trap) states and $\varepsilon_{i}$ be their energies. Take $i=0$ to be the lowest energy level, and take $\varepsilon_{i}=0$. In the presence of a chemical potential $\mu$, the mean occupation numbers $N_{i}$ for noninteracting bosons are

$$
N_{i}=\frac{1}{e^{\left(\varepsilon_{i}-\mu\right) / T}-1} .
$$

With the chosen zero of energy,

$$
e^{-\mu / T}=1+\frac{1}{N_{0}} \equiv \lambda^{-1}
$$

(defining the fugacity $\lambda$, to be used later). Once $N_{0} \gg 1$ (which may still be for $N_{0} \ll N$ ), the explicit fixed- $T N_{0}$ dependence of $N_{i>0}$ is $O\left(1 / N_{0}\right)$. The expression for the expected total number of particles with $i>0, N_{e}$, and how it depends on $\mu$ is determined by the density of states. For an isotropic harmonic oscillator potential in three dimensions with level spacing $\epsilon$,

$$
N_{e}=\zeta(3)(T / \epsilon)^{3}
$$

as long as $N_{e}<N$ and $T / \epsilon \gg 1[8]$. Under the latter condition, the asymptotic behavior of the sum over states is given by an integral. [ $\zeta(3) \approx 1.202$ is the Riemann zeta function.] Under these circumstances, the fixed- $T$ corrections to Eq. (3) are $O\left(1 / N_{0}\right)$. The root-mean-square fluctuation of any occupation number is precisely

$$
\Delta N_{i}=\sqrt{N_{i}\left(N_{i}+1\right)} .
$$

For the isotropic oscillator, this implies

$$
\Delta N_{e}=\sqrt{\frac{\pi^{2}}{6}(T / \epsilon)^{3}}
$$

so $\Delta N_{e} / N_{e} \sim O\left(1 / \sqrt{N_{e}}\right)$. The corrections to Eq. (5) for $\mu$ not exactly zero are again $O\left(1 / N_{0}\right)$.

The success of using a $\mu$ to characterize a system with a large but fixed total number of particles $N$ relies on the fact that each individual energy level is a system in diffusive equilibrium with the much larger remainder of the total system. This remainder acts as the single level's particle reservoir. Once $N_{0}$ is not much less than $N$, the utility of $\mu$ is no longer clear. Certainly there exists no yet-much-larger particle reservoir for the ground state.

Referring back to Eq. (1), once $N_{0}$ is large, the only role of the particular value of $\mu$ is to determine $N_{0}$. The $N_{i>0}$ are insensitive to $\mu$ or $N_{0}$. So, if we consider each individual excited level with $i>0$ as a system in contact with the reservoir of all the other levels, we need not know exactly what the chemical potential actually is, only that it is nearly zero. In fact, there need not be any precise meaning to $\mu$, only that it is nearly zero. It may be impossible to disentangle the effects of " $\mu \neq 0$ " from other $1 / N$ consequences of fixing the total $N$. From this perspective, $N_{0}$ is determined not by a $\mu$ but by $N$ and $N_{e}$ :

$$
N_{0}=N-N_{e} .
$$

However, this is precisely the same value of $N_{0}$ that is deduced from Eq. (1) when $N$ is interpreted as an expectation in the presence of an external $\mu$.

At the level of occupation expectations, the assignments given by Eq. (1) for $i>0$ minimize the total free energy (energy minus $T \times$ entropy) irrespective of the actual value of 
$N_{0}$ or $N$ as long as $N_{e}$ is fixed. This is because adding or removing particles from the $i=0$ condensate changes neither the energy nor the entropy of the entire system. Hence, for large $N_{0}$, the occupation numbers for $i>0$ are unchanged from their grand canonical values if, instead of being determined by a diffusive equilibrium, $N$ is fixed at some value and $N_{0}$ is large. Once there is a condensate, the only thing that can change as particles are added at fixed $T$ is $N_{0}$.

The total expected energy $\langle E\rangle$ at fixed $T$ depends only on the $i>0$ occupations. Thus canonical and grand canonical evaluations of the total energy must agree as $N \rightarrow \infty$. For the isotropic harmonic trap,

$$
\langle E\rangle=\frac{\pi^{4}}{30} T^{4} \epsilon^{-3}=\frac{\pi^{4}}{30 \zeta(3)} T N_{e} .
$$

Since it is a canonical ensemble identity that the root-meansquare total energy fluctuation satisfies

$$
\Delta E=\sqrt{T^{2} \frac{\partial\langle E\rangle}{\partial T}}
$$

the equivalence of the canonical and microcanonical ensembles is assured as long as $N_{e} \rightarrow \infty$ because $\Delta E / E \sim O\left(1 / \sqrt{N_{e}}\right)$. (This is true for any trapping potential, not just the explicit example given.)

\section{FLUCTUATION ESTIMATES}

From the discussion above, it is expected that all occupations approach their grand canonical values as $N \rightarrow \infty$, even if either or both $N$ and $E$ are fixed. One can go further and estimate the leading behavior of various quantities that vanish in this limit. As examples I consider the condensate fluctuations and the occupation correlations between levels.

As long as $N_{0} \ll N$, the root-mean-square fluctuation in the condensate number, $\Delta N_{0}$, satisfies Eq. (4). Once $N_{0} \sim O(N)$, Eq. (6) implies

$$
\Delta N_{0}=\Delta N_{e} .
$$

The crossover between these two behaviors is an example of the phenomena that make a direct analysis of the fixed- $N$ partition function difficult. It is appropriate to introduce the "critical" temperature $T_{c}$, given by the point at which $N_{e}$ reaches $N$ or, rather, at which $N_{0}$ goes from macroscopic to microscopic. For the isotropic harmonic potential, Eq. (3) implies

$$
T_{c}=N^{1 / 3} \zeta(3)^{-1 / 3} \epsilon .
$$

As $N$ increases, $T_{c}$ remains fixed in absolute, physical units only if the trap size is increased, e.g., $\epsilon$ decreased. The transition occurs when the central density in the trap reaches the infinite volume critical value [9]. In terms of the natural temperature variable for the study of Bose-Einstein condensation, $T / T_{c}$, the transition between Eq. (4) and Eq. (9) takes place in a vanishingly small interval as $N \rightarrow \infty$.

In the thermodynamic limit with $N, N_{0}$, and $N_{e}$ all very large, Eqs. (3), (5), (6), (9), and (10) can be combined to give a simple estimate of the leading behavior:

$$
\frac{\Delta N_{0}}{N_{0}}=\frac{1}{\sqrt{N}} \frac{\left(T / T_{c}\right)^{3 / 2}}{1-\left(T / T_{c}\right)^{3}}\left(\frac{\pi^{2}}{6 \zeta(3)}\right)^{1 / 2} .
$$

A set of quantities that are of interest in the calculation of the angular dependence of light scattering off cold, trapped atoms [10] are the two-level occupation expectations, $\left\langle n_{i} n_{j}\right\rangle$. (I use the notation $n_{i}$ for the actual $i$ th level occupation number in a particular configuration of the thermal ensemble.) In the grand canonical analysis of an ideal Bose gas, these are given precisely by $N_{i} N_{j}$. In particular, there is no correlation between the fluctuations in one level and another. However, with $N$ fixed, this cannot be exactly true. A refinement of the argument of the previous section allows one to estimate the leading behavior of these correlations. As an example consider the two states with the largest fluctuations, $i=0$ and $j=1$, because their fixed- $N$ induced correlation must, therefore, be the largest.

At fixed $N$, if $n_{0}$ fluctuates down, say, then $n_{e}$ must fluctuate up by an equal amount. The impact on the $n_{i>0}$ can be estimated by computing the particular expected $N_{i}$ given that $N_{e}$ is larger than its original equilibrium value by the negative of the $i=0$ fluctuation. This implies (writing $\Delta n_{i}$ for $\left.n_{i}-\left\langle n_{i}\right\rangle\right)$

$$
\left\langle\Delta n_{0} \Delta n_{1}\right\rangle=\left\langle\Delta n_{0}^{2}\right\rangle \frac{\delta\left\langle n_{1}\right\rangle}{\delta\left\langle n_{0}\right\rangle}=\left\langle\Delta n_{0}^{2}\right\rangle\left(-\frac{d N_{1} / d \lambda}{d N_{e} / d \lambda}\right)_{\lambda=1} .
$$

The fugacity $\lambda$ is defined by Eq. (2). For the isotropic, harmonic trap in the thermodynamic limit, this can be evaluated to give (with the natural normalization factor $N_{0} N_{1}$ )

$$
\begin{gathered}
\frac{\left\langle\Delta n_{0} \Delta n_{1}\right\rangle}{N_{0} N_{1}}=-N^{-2 / 3} \frac{T / T_{c}}{1-\left(T / T_{c}\right)^{3}} \zeta(3)^{-1 / 3} . \\
\text { V. NUMERICAL EVALUATION } \\
\text { OF THE CANONICAL ENSEMBLE }
\end{gathered}
$$

The canonical partition function $Z(N, T)$ of a trapped, ideal Bose gas can be represented as

$$
Z(N, T)=\frac{1}{2 \pi} \int_{-\pi}^{\pi} d z e^{i N z} \prod_{m=0}^{\infty} \sum_{n_{m}=0}^{\infty} e^{-n_{m} \varepsilon_{m} / T-i n_{m} z},
$$

where $n_{m}$ is the number of particles in the state labeled by $m$ with energy $\varepsilon_{m}$. The integral over $z$ implements the constraint $N=\Sigma_{m} n_{m}$. For the isotropic harmonic potential in three dimensions, it is convenient to let $m$ label the energy levels $\varepsilon_{m}=m \epsilon$, with the associated degeneracy of $\frac{1}{2}(m$ $+1)(m+2)$ for $m=0,1,2, \ldots$ The infinite sums over occupations can be done explicitly. Occupation expectations and correlations can be represented similarly by simple modifications of the integrand, i.e., extra weight factors of $n_{i}$ or $n_{i} n_{j}$. If one truncates the infinite product over energy levels $m$ at some finite $M_{\max }$, this yields a form that can be evaluated numerically. One can study the convergence in $M$ to test whether the asymptotic values of thermal expectations have plausibly been reached. (Useful numerical strategies and some details of the evaluations are provided in Appendix A.) 


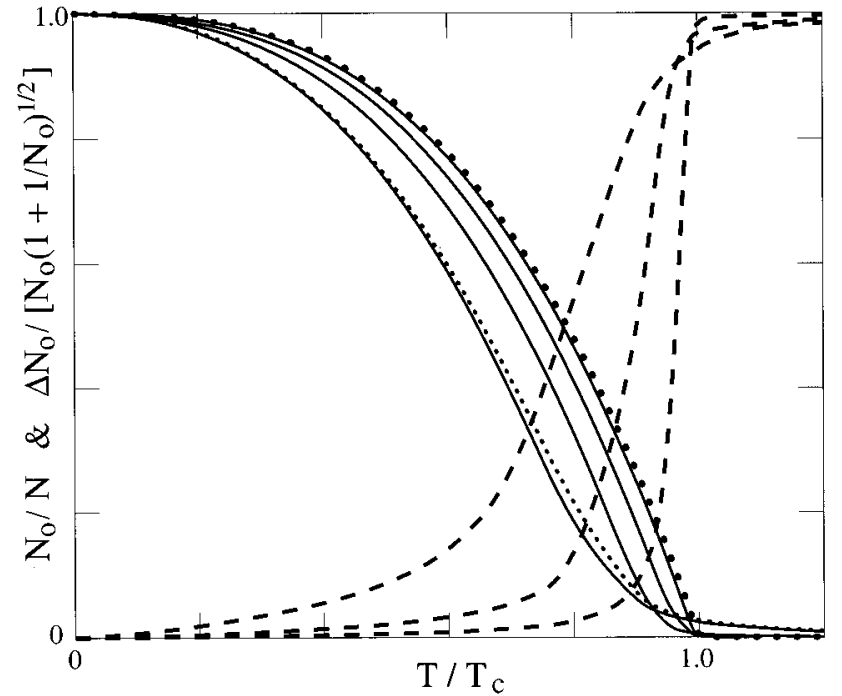

FIG. 1. Canonical $N_{0} / N$ for $N=10^{2}, 10^{3}, 10^{4}$, and $10^{6}$ (solid lines), grand canonical $N_{0} / N$ for $N=10^{2}$ (small dots), the grand canonical $N \rightarrow \infty$ limit (large dots), and the normalized canonical condensate rms fluctuations (dashed lines) for $N=10^{2}, 10^{3}$, and $10^{4}$ vs $T / T_{c}$.

Figure 1 shows the results of calculations of $N_{0}$. In particular, the solid lines are the numerically computed values of $N_{0} / N$ for $N=10^{2}, 10^{3}, 10^{4}$, and $10^{6}$, plotted versus $T / T_{c}$, where $T_{c}$ is given by Eq. (10) appropriate to each $N$. The dotted lines are grand canonical predictions for $N=10^{2}$ (small dots) and the $N \rightarrow \infty$ limit, $1-\left(T / T_{c}\right)^{3}$ (large dots). Note that the grand canonical predictions were computed as sums over states using Eqs. (1) and (2) and involve no approximations regarding $N$. The comparison of the two statistical ensembles is made by identifying the value of the grand canonical $\langle N\rangle$ with the precise canonical $N$. The canonical numerical calculations clearly approach the $N \rightarrow \infty$ grand canonical form as a limiting value with increasing $N$. For intermediate values of $T / T_{c}$, e.g., 0.6, the fractional discrepancy between the canonical $N$ and $N \rightarrow \infty$, i.e., difference divided by value, appears to be decreasing roughly as $N^{-0.33}$.

The differences between canonical and grand canonical values for $N_{0}$ are displayed in another way in Fig. 2. The fractional discrepancy between the two evaluations is plotted for $N=10^{2}, 10^{3}$, and $10^{4}$ versus $T / T_{c}$. Here, "fractional discrepancy" means $\left(N_{0}^{\text {grand canonical }}-N_{0}^{\text {canonical }}\right) /$ $N_{0}^{\text {grand canonical }}$. At very small $T / T_{c}$, all evaluations give $N_{0} / N$ very near to 1 . So the ratio plotted in Fig. 2 plummets, but it is not an effective way to characterize the difference between fixed $N$ and fixed $\mu$. (For that region, a more informative variable would be $N_{1}$.) For intermediate values of $T / T_{c}$, the curves of Fig. 2 decrease roughly as $N^{-1.15}$. So, not only does the canonical $N_{0}$ approach $N\left[1-\left(T / T_{c}\right)^{3}\right]$ as $N \rightarrow \infty$, it does so approximately as predicted by the simple grand canonical calculation. It is the next correction, the difference between the two ensembles' predictions at a given $N$ (as illustrated in Fig. 2) that reflects the residual difference in physics between the ensembles. This difference is particularly pronounced as $N_{0}$ makes the transition from microscopic to macroscopic just below $T_{c}$. There, the grand

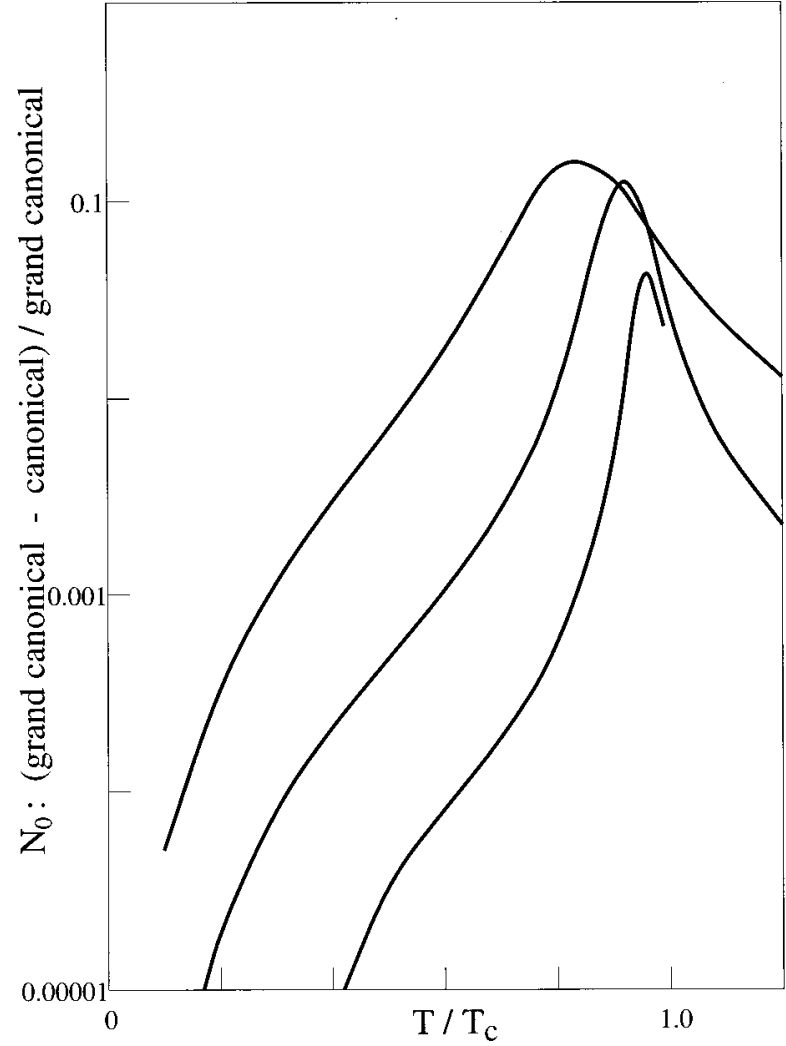

FIG. 2. Comparison of the canonical and grand canonical values for $N_{0}$ as fractional discrepancies on a logarithmic scale for $N=10^{2}, 10^{3}$, and $10^{4}$ vs $T / T_{c}$.

canonical-canonical discrepancy decreases only very slowly with $N$. The sign and shape of the difference are such that the canonical $N_{0}$ does not rise quite as sharply as the grand canonical, but the width of the relevant region of $T / T_{c}$ vanishes with increasing $N$. Above $T_{c}$, the distinction between fixing $N$ and fixing $\mu$ has rapidly vanishing consequences.

The dashed lines in Fig. 1 are the results of a numerical evaluation of the canonical $\Delta N_{0} /\left[N_{0}\left(N_{0}+1\right)\right]^{1 / 2}$ versus the same $T / T_{c}$ 's for $N=10^{2}, 10^{3}$, and $10^{4}$. For $T \gtrsim T_{c}$, this ratio approaches 1, in agreement with the grand canonical Eq. (4). However, for $T<T_{c}$, it goes to zero, more dramatically with increasing $N$. These same $\Delta N_{0}$ data are plotted again on a logarithmic scale as the solid lines in Fig. 3. The dotted lines are plots of Eq. (11) for the same values of $N$. As long as neither $N_{0}$ nor $N_{e}$ is too small, Eq. (11) clearly captures the $N$ and $T$ dependence of $\Delta N_{0}$, and the agreement improves with increasing $N$. In particular, the fractional discrepancy between the canonical and Eq. (11) values appears to go roughly as $N^{-0.25}$.

The canonical, normalized, fluctuation correlation, $-\left\langle\Delta n_{0} \Delta n_{1}\right\rangle / N_{0} N_{1}$, is plotted (solid lines) on a logarithmic scale versus $T / T_{c}$ for $N=10^{2}, 10^{3}$, and $10^{4}$ in Fig. 4. The overall minus sign is because the correlation is, indeed, negative. The dotted lines are Eq. (13) for the same three values of $N$, and again the agreement improves with $N$; this time the fractional discrepancy appears to go roughly as $N^{-0.33}$.

The discrepancies between the numerical evaluations and the simple formulas are largest for $T$ 's such that either $N_{0}$ or $N_{e}$ is not very large. These are vanishingly small intervals of $T / T_{c}$ for $N \rightarrow \infty$. 


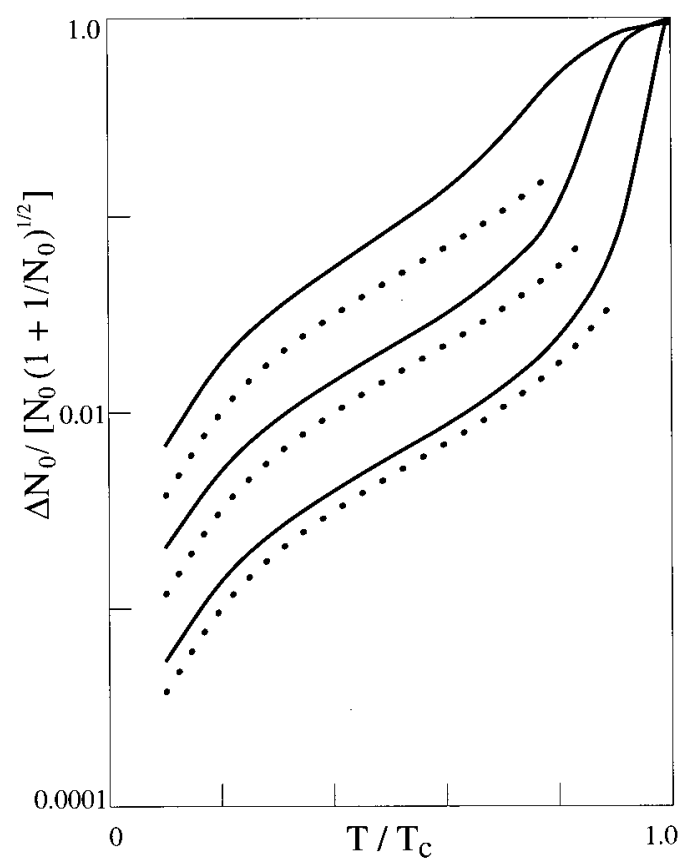

FIG. 3. A logarithmic plot of the canonical condensate rms fluctuations (solid lines) and the simple equation (11) estimates (dotted lines) for $N=10^{2}, 10^{3}$, and $10^{4}$.

The expected $i=1$ occupation, $N_{1}$, was evaluated to prepare Fig. 4. The agreement with Eq. (1) with $\mu=0$ was such that the leading fractional discrepancy was accounted for by just the leading $1 / N_{0}$ correction already included in Eq. (1), i.e., $T / N_{0}$.

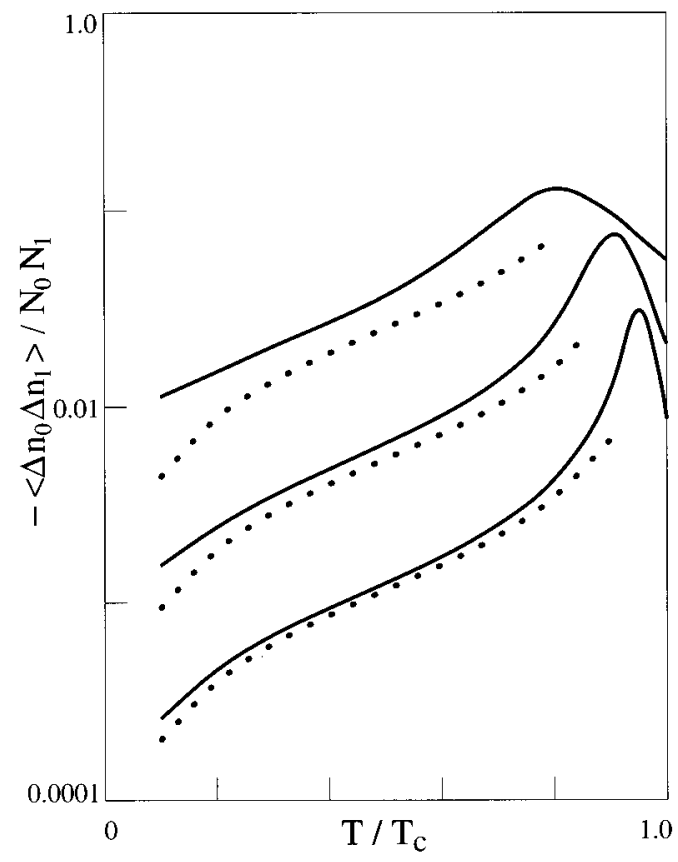

FIG. 4. A logarithmic plot of -1 times the normalized, canonical 0-1 level correlations (solid lines) and the simple equation (13) estimates (dotted lines) for $N=10^{2}, 10^{3}$, and $10^{4}$.
The particular computer code used for the results presented was checked against hand calculations for small $N$. For large $N$, a criterion for validity was stability under changes in the several parameters that should not effect the final answers. Eventually, at high enough $N$ (different values for different observables) the ranges of stability in these parameters shrunk to zero. Typically, the practical limitation was the digits of precision available for intermediate results. The code was written to evaluate $N_{0}$ below $T_{c}$, and specifically for $N_{0}$ plausible results were obtained for much higher $N$ than presented. No effort was made to modify the numerical strategy to facilitate calculation of the other quantities discussed; presumably those calculations could be extended to higher $N$ with algorithmic improvements that avoided the simultaneous evaluation of numbers of vastly different magnitudes.

\section{DISCUSSION AND CONCLUSIONS}

The general arguments presented here, while heuristic, have an internal consistency. For example, to compute $\Delta N_{e}$, which is used implicitly in Eqs. (12) and (13), one assumes that the correlations between level occupations are negligible. One then deduces nonzero correlations that are induced by particle conservation. However, the induced correlations are, indeed, small enough to be ignored in the calculation of the leading behavior of $\Delta N_{e}$ and of the correlations themselves.

This is nowhere near to a "theory" of the large- $N$ asymptotics of the canonical ideal Bose gas. The leading behavior of some interesting observables was estimated and confirmed numerically. But in these cases, the leading behavior either was simply given by or could be deduced from the grand canonical ensemble. The next level of approximation, e.g., to account for Fig. 2, would require a detailed analysis of the canonical or microcanonical partition function and may be very difficult to determine analytically.

Starting with the grand canonical description with $\mu$ and $T$ as independent variables, one finds large fluctuations in $N$ below $T_{c}$. Hence, fixing $N$ may have been expected to be of some consequence. However, the grand canonical total energy fluctuations are always small and vanish relative to the mean total energy in the thermodynamic limit. Nothing special happens in $E$ at $T_{c}$. So fixing $E$ should have no dramatic consequences. Overall, the switch from $T$ to $E$ should be of even less consequence than the switch from $\mu$ to $N$. A direct numerical evaluation of the microcanonical partition function would be considerably more difficult.

However, from a practical standpoint, the modest results here are useful. The largest consequence of going from a chemical potential to fixed $N$ for an ideal Bose gas is that the ground-state number fluctuations are always microscopic; the leading behavior of all expected level occupations is unchanged. This is sufficient to further imply that fixing the total $E$ is no different from the analytically simpler fixing of $T$ in the thermodynamic limit. The leading behaviors of the two-level expectations, $\left\langle n_{i} n_{j}\right\rangle$ for $i \neq j$, are unchanged because the induced correlations vanish as $N \rightarrow \infty$. For large, fixed $N$, the corrections to these behaviors are unlikely to be of any practical importance. As discussed in Appendix B, for a gas with replusive interactions, the consequence of fixing 
$N$ dominates over the interaction effects in damping the ground-state number fluctuations only if the pairwise energy in the ground state is less than $O\left(N^{-2 / 3} \epsilon\right)$.

\section{ACKNOWLEDGMENTS}

Werner Krauth of ENS, Paris, pointed out that an earlier effort along these lines was in error and suggested the numerical strategy followed here. Anton Kapustin patiently offered suggestions and criticism. This work was supported in part by the U.S. Department of Energy under Grant No. DEFG03-92-ER40701.

\section{APPENDIX A: NUMERICAL STRATEGIES}

For the isotropic harmonic potential in three dimensions and a maximum energy level $M_{\max }$, Eq. (14) takes the explicit form

$$
\begin{aligned}
Z(N, T)= & \frac{1}{2 \pi} \int_{-\pi}^{\pi} d z e^{i N z} \\
& \times \prod_{m=0}^{M_{\max }}\left(\frac{1}{1-e^{-m \epsilon / T-i z}}\right)^{(1 / 2)(m+1)(m+2)} .
\end{aligned}
$$

A rather primitive C program on a Sun SPARC10 for integrating Eq. (A1) and related functions was sufficient to generate the numerical results presented in the figures, with the size of $N$ limited by the use of double-precision arithmetic. A few general observations may prove to be of some value in any future effort to perform comparable calculations.

Instead of simply truncating the product over energy levels $m$ at some large value $M_{\max }$, one can use MaxwellBoltzmann statistics for all levels $m>M_{\max }$ and derive an approximate closed form for the contribution to the integrand of all levels above $M_{\max }$. This vastly improves the rate of convergence in $M_{\max }$ because for modest $m$ 's (e.g., 6T/ $\epsilon$ ) there are still quite a few particles at that $m$ or higher, but the occupations of individual states are rarely greater than 1 .

By far the most rapid variation of the integrand for large $N$ comes from the factor $e^{i N z}$. The integration algorithm should reflect this knowledge. For example, one can divide $z$ into intervals of $\pi / 4 N$ and integrate each interval accordingly. (For the largest values of $N$ it proved sufficient to take a single point in each such interval.)

An overall factor in $Z$ has no effect on physical observables. This can be used to considerable advantage. Here are a couple of examples: One can evaluate the products of very large numbers logarithmically, i.e., sum the phase and logarithm of the modulus of the various complex factors. An overall shift before exponentiation and addition (integration) keeps numbers from getting too big. Also, observables are independent of shifts of the whole energy spectrum by the ground-state energy $\varepsilon_{0}$. It is convenient to take this nonzero to check the numerical independence. Taking $\varepsilon_{0} \neq 0$ can also dramatically alter the character of the integrand of Eq. (A1) - note the (analytically integrable) singularity at $z=0$ for $\varepsilon_{0}=0$.

It is, of course, sufficient to integrate only $0 \leqslant z \leqslant \pi$. With suitable choice of $\varepsilon_{0}$, starting at $z=0$ one can integrate outward, test the convergence, and exit the integration long before reaching $z=\pi$.

\section{APPENDIX B: INTERACTION DAMPING OF GRAND CANONICAL OCCUPATION FLUCTUATIONS}

The effect of a weak repulsive interaction on condensate fluctuations can be estimated as follows. Let $n_{0}$ represent the number of particles in the ground state. The leading effect of a weak, pairwise repulsion at low $T$, when most of the particles are in the ground state, is to raise the energy of those particles from 0 (a convenient $n$-independent normalization of the noninteracting ground-state energy) to $\lambda n_{0}^{2}$, where $\lambda$ is the positive two-particle interaction contribution to the ground-state energy. In natural oscillator units ( $\left.\hbar=m=\omega_{0}=1\right), \lambda$ is related to the conventionally defined scattering length $a$ by $\lambda=a / \sqrt{2 \pi}$, at least if the interaction effects are weak enough to be treated in mean-field theory. Focus on the terms in the grand canonical partition function that refer only to the ground state:

$$
\mathcal{Q}_{0}(\mu, T)=\sum_{n_{0}=0}^{\infty} e^{\mu n_{0} / T-\lambda n_{0}^{2} / T} .
$$

Unlike the $\lambda=0$ case, one can now get large $N_{0}\left(=\left\langle n_{0}\right\rangle\right)$ with $\mu>0$. Then, the sum can be considered as an integral over $n_{0}$, whose integrand is a Gaussian peaked at $n_{0}=N_{0}=\mu / 2 \lambda \quad$ with width $\Delta N_{0}=\sqrt{T / \lambda}$. Hence, $\Delta N_{0} / N_{0}=\sqrt{T /\left(\lambda N_{0}^{2}\right)}$, in contrast to the $\lambda=0$ situation, in which $\Delta N_{0} / N_{0}$ is $O(1)$. So grand canonical condensate number fluctuations are small if the interaction contribution to the ground-state energy is large compared to the temperature.

If sufficiently strong, interatomic repulsion will certainly be effective at damping condensate fluctuations at fixed $N$, giving $\Delta N_{0} \sim \sqrt{T / \lambda}$. This effect will dominate (i.e., enforce smaller fluctuations) over the noninteracting $\Delta N_{0} \sim \sqrt{(T / \epsilon)^{3}}$ estimated in Secs. III and IV when $\lambda / \epsilon \gtrsim(T / \epsilon)^{-2}$. ( $\epsilon$ is the trap level spacing, and $T_{c} / \epsilon \sim N^{1 / 3}$.)
[1] See, e.g., C. Kittel and H. Kroemer, Thermal Physics, 2nd ed. (Freeman, San Francisco, 1980).

[2] See, e.g., K. Huang, Statistical Mechanics, 2nd ed. (Wiley, New York, 1987).

[3] See, e.g., M. Rasolt, M.J. Stephen, M.E. Fisher, and P.B. We- ichman, Phys. Rev. Lett. 53, 798 (1984); P.B. Weichman, Phys. Rev. B 34, 7652 (1984).

[4] M.H. Anderson, J.R. Ensher, M.R. Matthews, C.E. Wieman, and E.A. Cornell, Science 269, 198 (1995).

[5] C.C. Bradlet, C.A. Sackett, J.J. Tollett, and R.G. Hulet, Phys. 
Rev. Lett. 75, 1687 (1995).

[6] K.B. Davis, M.O. Mewes, M.R. Andrews, N.J. van Druten, D.S. Durfee, D.M. Kurn, and W. Ketterle, Phys. Rev. Lett. 75, 3969 (1995).

[7] F. London, Phys. Rev. 54, 947 (1938).
[8] S.A. de Groot, G.J. Hooyman, and A. Sedlam, Proc. R. Soc. London Ser. A 203, 266 (1950).

[9] V. Bagnato, D.E. Pritchard, and D. Kleppner, Phys. Rev. A 35, 4354 (1987).

[10] H.D. Politzer, Phys. Lett. A 209, 160 (1995). 